\title{
APPENDICITIS AND ANTIBIOTICS AN APPROACH TO THE MANAGEMENT OF ACUTE APPENDICITIS
}

Sathish .V. Shivamallaiah ${ }^{1}$

\section{HOW TO CITE THIS ARTICLE:}

Sathish V. Shivamallaiah. "Appendicitis and Antibiotics an approach to the Management of Acute Appendicitis". Journal of Evolution of Medical and Dental Sciences 2014; Vol. 3, Issue 59, November 06; Page: 13282-13287, DOI: $10.14260 /$ jemds/2014/3768

ABSTRACT: In this era of rapid emergence of antibiotic resistant microorganisms it is advisable to use the antibiotics judiciously. It is a challenge to the medical researchers and clinicians to treat such cases and in large protect the future generation from dreaded diseases because of these microorganisms. Since appendicitis is the most common surgical emergency throughout the globe it is convenient and prudent to take up appendicitis for this sort of study. $(1,2,3)$

KEYWORDS: Appendicitis, Clean non-perforated, Antibiotic resistance.

INTRODUCTION: In this era of growth of antibiotic resistant microbes, a study was conducted in patients with acute appendicitis, where a single dose of antibiotic prior to operation (ceftriaxone $1 \mathrm{gm}$ ) to all patients and continued in those with pus (post-operative) and withholding the antibiotic in clean non-perforated cases.

MATERIALS \& METHODS: All patients with appendicular mass and patients with ultrasound proven pelvic inflammatory diseases were excluded from the study. All patients diagnosed with appendicitis, had undergone open appendectomy irrespective of size of appendix and all age group patients were included from $1^{\text {st }}$ of January to 31 $1^{\text {st }}$ December 2013 in our hospital. In all cases the outcome of surgery in terms of time taken for recovery from the illness, wound infection, duration of hospital stay and duration of antibiotic usage were studied and results were compared.

OBSERVATION \& INFERENCE: Out of 75 patients diagnosed with appendicitis on ultrasound abdomen irrespective of size of appendix, were given single dose of intravenous ceftriaxone $1 \mathrm{mg}$ and taken up for surgery after routine blood investigation and physician fitness. On operation 66 patients were having clean, non-perforated appendicitis without pus intra-peritonealy, out of which 34 patients were given antibiotics for 3 days (group A) and 32 patients were not given antibiotics after operation (group B) rest 9 patients (out of which 2 had appendicular abscess and 3 had appendicular mass and 4 had pus in right iliac fossa) were excluded from the study and were managed expectantly.

For all patients included in the study alternate day dressing was done. Out of 32 patients in group B (only pain killers aceclofenac $100 \mathrm{mg}$ on demand was given and allowed orally as soon as bowel sounds appeared) 6 patients had wound infection, in all cases pus was sensitive to ofloxacin and cefixime, which were given orally for 5 days and daily dressing done for 5 days and alternate day dressing for next one week and wound healed within 2 weeks, these patients hospital stay was not extended because of the wound infection. On average group B patients stayed in the hospital for 3 days.

Out of 34 patients in group A 4 patients had wound infection, out of which 3 patient's pus grown E. Coli and sensitive for ciprofloxacin, which was given orally for 7 days and remaining 


\section{ORIGINAL ARTICLE}

patient's pus was sensitive for amoxicillin-clavulinic acid which was given orally for 7 days. Wound care was given to these patients similar to group B.

\begin{tabular}{|c|c|c|c|}
\hline & Wound Infection & \multirow{2}{*}{ Total } \\
\cline { 2 - 3 } & Present & Absent & \\
\hline Group A & 4 & 30 & 34 \\
\hline Group B & 6 & 26 & 32 \\
\hline Total & $\mathbf{1 0}$ & $\mathbf{5 6}$ & $\mathbf{6 6}$ \\
\hline \multicolumn{3}{|c|}{ Table 1 } \\
\hline
\end{tabular}

Odds Ratio $=0.5778$ [95\% CI: $0.1468-2.2734]$.

Risk Ratio $=0.6275$ [95\%CI: $0.1948-2.0205]$.

$P$ value (Fisher Exact Probability Test) $=0.5052$.

CONCLUSION: Even without regular antibiotics, operated appendicitis cases were treated satisfactorily with regular dressings and painkillers and also with less usage of intravenous antibiotics and can be treated without much of morbidity hence this study recommends judicious and careful use of antibiotics to avoid rapid increase of antibiotic resistance microbes.

DISCUSSION: Appendicitis is a common surgical condition across the world and affects nearly $40 \%$ of world's population in their life time. Appendix is a derivative of mid gut and it varies in position which reflects its varying clinical presentation and diagnostic confusions. Credit for performing the first appendectomy goes to Claudius Amyand, a surgeon at St. George's Hospital in London and Sergeant Surgeon to Queen Ann, King George I, and King George II. In 1736, he operated on an 11year-old boy with a scrotal hernia and a fecal fistula. Within the hernial sac, Amyand found the appendix perforated by a pin. He successfully removed the appendix and repaired the hernia. $(2,3)$

In 1824, Louyer-Villermay presented a paper before the Royal Academy of Medicine in Paris. He reported on two autopsy cases of appendicitis and emphasized the importance of the condition. In 1827, François Melier, a French physician, expounded on Louyer-Villermay's work. He reported six autopsy cases and was the first to suggest the antemortem recognition of appendicitis. This work was discounted by many physicians of the era, including Baron Guillaume Dupuytren. Dupuytren believed that inflammation of the cecum was the main cause of pathology of the right lower quadrant.

In 1839, a textbook by Bright and Addison entitled Elements of Practical Medicine described the symptoms of appendicitis and identified the primary cause of inflammatory processes of the right lower quadrant. Reginald Fitz, is credited with coining the term appendicitis. Appendicitis is an outcome of obstruction of the lumen of the appendix, which leads to increase in its luminal pressure and the clinical symptoms. The causes for appendicitis rather, obstruction of appendicular lumen is inspisated stool called fecolith or appendicolith, lymphoid hyperplasia, vegetable matter or seeds, parasites or neoplasm. $(1,2,3,4,5)$

The lumen of the appendix is small in relation to its length, and this configuration may predispose to closed-loop obstruction. Obstruction of the appendiceal lumen contributes to bacterial overgrowth, and continued secretion of mucus leads to intraluminal distention and increased wall pressure, producing visceral pain sensation experienced by the patient as periumbilical pain. 


\section{ORIGINAL ARTICLE}

Subsequent impairment of lymphatic and venous drainage leads to mucosal ischemia, promoting inflammatory process that may progress to gangrene and perforation.

Inflammation of the adjacent peritoneum gives rise to localized pain in the right lower quadrant. Perforation typically occurs after at least 48 hours from the onset of symptoms and forms an abscess cavity walled-off by the small intestine and omentum. Rarely, free perforation of the appendix into the peritoneal cavity occurs that may be accompanied by peritonitis and septic shock with subsequent formation of multiple intraperitoneal abscesses. The polymicrobial nature of perforated appendicitis is well established as its flora is similar to that of colon.

Escherichia coli, Streptococcus viridans, and Bacteroides and Pseudomonas species are frequently isolated, and many other organisms may be cultured. Among patients with acute nonperforated appendicitis, cultures of peritoneal fluid are frequently negative and are of limited use. Among patients with perforated appendicitis, peritoneal fluid cultures are more likely to be positive, revealing colonic bacteria with predictable sensitivities. Because it is rare that the findings alter the selection or duration of antibiotic use, some authors have challenged the traditional practice of obtaining cultures. $(2,3,4,5,1)$

SYMPTOMS AND SIGHNS: Anatomic variations in the position of the inflamed appendix lead to deviations in the usual physical findings. Vital signs are minimally changed by uncomplicated appendicitis. Temperature elevation is rarely $>1^{\circ} \mathrm{C}\left(1.8^{\circ} \mathrm{F}\right)$ and the pulse rate is normal or slightly elevated. Patients with appendicitis usually prefer to lie supine, with the thighs, particularly the right thigh, drawn up, because any motion increases pain. If asked to move, they do so slowly and with caution.

Tenderness is often maximal at or near the McBurney's point when the inflamed appendix lies in the anterior position. Direct rebound tenderness usually is present. In addition, referred or indirect rebound tenderness is present. This referred tenderness is felt maximally in the right lower quadrant, which indicates localized peritoneal irritation. The Rovsing sign-pain in the right lower quadrant when palpatory pressure is exerted in the left lower quadrant-also indicates the site of peritoneal irritation.

Cutaneous hyperesthesia in the area supplied by the spinal nerves on the right at $\mathrm{T} 10, \mathrm{~T} 11$, and T12 frequently accompanies acute appendicitis. In patients with obvious appendicitis, this sign is superfluous, but in some early cases, it may be the first positive sign. Guarding at the RIF roughly parallels the severity of the inflammatory process, is present in early disease as peritoneal irritation progresses, involuntary skeletal muscle spasm sets in leading to rigidity. Perrectal examination helps in diagnosis of pelvic appendicitis. As the examining finger exerts pressure on the peritoneum of Douglas' cul-de-sac, pain is felt in the suprapubic area as well as locally within the rectum.

Signs of localized muscle irritation also may be present. The psoas sign indicates an irritative focus in proximity to that muscle. The test is performed by having the patient lie on the left side and slowly extend the patient's right thigh, thus stretching the iliopsoas muscle. The test result is positive if extension produces pain. Similarly, a positive obturator sign of hypogastric pain on stretching the obturator internus indicates irritation in the pelvis which is performed by passive internal rotation of the flexed right thigh with the patient supine. $(1,2,3,4$, 


\section{ORIGINAL ARTICLE}

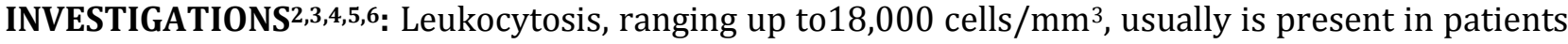
with acute, uncomplicated appendicitis and often is accompanied by a moderate polymorph nuclear predominance. White blood cell counts above this level raise the possibility of a perforated appendix with or without an abscess. Urinalysis can be useful to rule out the urinary tract as the source of infection. A plain abdominal radiograph in the evaluation of patients with acute abdominal pain is unwarranted. A calcified appendicolith is visible on plain films in only $10 \%$ to $15 \%$ of patients with acute appendicitis; however, its presence strongly supports the diagnosis in a patient with abdominal pain.

BARIUM ENEMA: Failure of the appendix to fill during a barium enema has been associated with appendicitis, but this finding lacks both sensitivity and specificity because up to $20 \%$ of normal appendices do not fill.

ULTRASONAGRAPHY: It has a sensitivity of about $85 \%$ and a specificity of more than $90 \%$ for the diagnosis of acute appendicitis. Sonographic findings consistent with acute appendicitis include an appendix of $7 \mathrm{~mm}$ or more in anteroposterior diameter, a thick-walled, non-compressible luminal structure seen in cross section referred to as a target lesion, or the presence of an appendicolith. In more advanced cases, periappendiceal fluid or a mass may be found.

Ultrasonography, being a noninvasive modality, avoids exposure to ionizing radiation. Disadvantages of ultrasonography include operator-dependent accuracy and difficulty interpreting the images by those other than the operator. Pelvic ultrasound can be especially useful in excluding pelvic pathology, such as tubo-ovarian abscess or ovarian torsion that may mimic acute appendicitis

COMPUTED TOMOGRAPHY (CT) SCAN: This scan has a sensitivity of about $90 \%$ and a specificity of $80 \%$ to $90 \%$ in the diagnosis of acute appendicitis using $5 \mathrm{~mm}$ section. Contrast studies in the diagnosis of acute appendicitis are controversial in general, CT findings of appendicitis increase with the severity of the disease. Classic findings include a distended appendix greater than $7 \mathrm{~mm}$ in diameter and circumferential wall thickening, which may give the appearance of a halo or target.

As inflammation progresses, one may see periappendiceal fat stranding, edema, peritoneal fluid, phlegmon, or a periappendiceal abscess. CT detects appendicoliths in about $50 \%$ of patients with appendicitis and also in a small percentage of people without appendicitis. Among patients with abdominal pain, the positive predictive value of the finding of an appendicolith on CT remains high at about $75 \%$.

DIAGNOSTIC LAPAROSCOPY: Although most patients with appendicitis will be accurately diagnosed based on history, physical exam, laboratory studies, and if necessary, imaging techniques, there are a small number in whom the diagnosis remains elusive where diagnostic laparoscopy can provide both a direct examination of the appendix and a survey of the abdominal cavity for other possible causes of pain. Concerns about the possible adverse effects of a missed perforation and peritonitis on future fertility sometimes prompt earlier intervention in this patient population. $(2,3,4,5,6)$

DIFFERENTIAL DIAGNOSIS: Various conditions according to age and sex should be considered in the course of diagnosis of appendicitis which includes, renal calculi, ureteric calculi, ovarian torsion, ectopic pregnancy, tubal rupture etc. $(1,2,3)$ 


\section{ORIGINAL ARTICLE}

TREATMENT(2,3,5,6): Initial surgical therapy for appendicitis was primarily designed to drain right lower quadrant abscesses that occurred secondary to appendiceal perforation. It appears that the first surgical treatment for appendicitis or perityphlitis without abscess was carried out by Hancock in 1848. He incised the peritoneum and drained the right lower quadrant without removing the appendix. The first published account of appendectomy for appendicitis was by Krönlein in 1886.Fergus, in Canada, performed the first elective appendectomy in 1883.

The greatest contributor to the advancement in the treatment of appendicitis was Charles McBurney. In 1889, he published his landmark paper in the New York State Medical Journal describing the indications for early laparotomy for the treatment of appendicitis. It is in this paper that he described the McBurney point as follows: "maximum tenderness, when one examines with the fingertips is, in adults, one half to two inches inside the right anterior spinous process of the ilium on a line drawn to the umbilicus."

McBurney subsequently published a paper in 1894 describing the incision that bears his name. However, McBurney later credited McArthur with first describing this incision. Semm is widely credited with performing the first successful laparoscopic appendectomy in 1982.The surgical treatment of appendicitis is one of the great public health advances of the last 150 years.

Appendectomy for appendicitis is the most commonly performed emergency operation in the world. Appendicitis is a disease of the young, with $40 \%$ of cases occurring in patients between the ages of 10 and 29 years. In 1886, Fitz reported the associated mortality rate of appendicitis to be at least $67 \%$ without surgical therapy. Currently, the mortality rate for acute appendicitis with treatment is reported to be $<1 \%$.

Despite the advent of more sophisticated diagnostic modalities, the importance of early operative intervention should not be minimized. A large meta-analysis has demonstrated the efficacy of preoperative antibiotics in lowering the infectious complications in appendicitis. Most surgeons routinely administer antibiotics to all patients with suspected appendicitis. If simple acute appendicitis is encountered, there is no benefit in extending antibiotic coverage beyond 24 hours.

Some surgeons follow preoperative antibiotics cover aerobic and anaerobic colonic flora, for patients with non-perforated appendicitis, a single preoperative dose of antibiotic reduces postoperative wound infections and intra-abdominal abscess formation. Postoperative oral antibiotics do not further reduce the incidence of infectious complications in these patients.

For patients with perforated or gangrenous appendicitis, continue postoperative intravenous antibiotics until the patient is afebrile and has a normal white blood cell count. For intra-abdominal infections of GI tract origin that are of mild to moderate severity, the Surgical Infection Society has recommended single-agent therapy with cefoxitin, cefotetan, or ticarcillin-clavulanic acid.

For more severe infections, single-agent therapy with carbapenems or combination therapy with a third-generation cephalosporin, monobactam, or aminoglycoside plus anaerobic coverage with clindamycin or metronidazole is indicated. The recommendations are similar for children. $(2,3,5,6)$

CONCLUSION: To conclude, judicious use of antibiotics in appendicitis such as single dose, pre operatively in simple non-perforated cases and till febrile period in complicated cases will not cause increase in mortality or morbidity. 


\section{ORIGINAL ARTICLE}

\section{REFERENCES:}

1. Bailey \& Love's textbook of surgery 23rd edition, page no: 885-890.

2. Sabiston's textbook of surgery $18^{\text {th }}$ edition vol-2, page no $1333-1345$.

3. Schwartz's textbook of surgery 9th edition, page no: 1073-1089.

4. Harrison's textbook of surgery $16^{\text {th }}$ edition, page no: $1805-1809$.

5. Mangoits textbook of abdominal surgeries $1^{\text {st }}$ volume 1023.

6. Wikipedia free encyclopedia internet reference.

\section{AUTHORS:}

1. Sathish V. Shivamallaiah

\section{PARTICULARS OF CONTRIBUTORS:}

1. Assistant Professor, Department of General Surgery, Kodagu Institute of Medical Sciences, Madikeri, Kodagu District.

\section{NAME ADDRESS EMAIL ID OF THE} CORRESPONDING AUTHOR:

Dr. Sathish V. Shivamallaiah, \# 61, New KHB Ooty Road,

Nanjangud,

Mysore District.

Email: dr.sathish.vs@gmail.com

Date of Submission: 22/10/2014. Date of Peer Review: 23/10/2014. Date of Acceptance: 30/10/2014. Date of Publishing: 05/11/2014. 